\title{
Microsaccades and Blinks Trigger Illusory Rotation in the "Rotating Snakes" Illusion
}

\author{
Jorge Otero-Millan, ${ }^{1,2}$ Stephen L. Macknik, ${ }^{1}$ and Susana Martinez-Conde ${ }^{1}$ \\ ${ }^{1}$ Barrow Neurological Institute, Phoenix, Arizona 85013, and ${ }^{2}$ University of Vigo, 36310 Vigo, Spain
}

Certain repetitive arrangements of luminance gradients elicit the perception of strong illusory motion. Among them, the "Rotating Snakes Illusion" has generated a large amount of interest in the visual neurosciences, as well as in the public. Prior evidence indicates that the Rotating Snakes illusion depends critically on eye movements, yet the specific eye movement types involved and their associated neural mechanisms remain controversial. According to recent reports, slow ocular drift-a nonsaccadic type of fixational eye movement-drives the illusion, whereas microsaccades produced during attempted fixation fail to do so. Here, we asked human subjects to indicate the presence or absence of rotation during the observation of the illusion while we simultaneously recorded their eye movements with high precision. We found a strong quantitative link between microsaccade and blink production and illusory rotation. These results suggest that transient oculomotor events such as microsaccades, saccades, and blinks, rather than continuous drift, act to trigger the illusory motion in the Rotating Snakes illusion.

\section{Introduction}

The "Rotating Snakes Illusion" produces the perception of strong illusory rotation in most observers and has generated an extraordinary amount of academic and popular interest (Fig. 1) (Martinez-Conde and Macknik, 2010; Ramachandran and Rogers-Ramachandran, 2010). Here we demonstrate a direct relationship between the onsets of both microsaccades and blinks and perceived illusory rotation, showing that transient oculomotor events act to trigger the Rotating Snakes effect.

More than three decades ago, Fraser and Wilcox (1979) developed a repetitive spiral arrangement of sawtooth luminance gradients that elicited illusory motion (Fig. 1A). The "Peripheral Drift Illusion"- a variant on the Fraser-Wilcox effect-later incorporated circular rather than spiral arrangements of sawtooth luminance gradients (Fig. 1B) (Faubert and Herbert, 1999). Kitaoka and Ashida (2003) subsequently developed a new and improved version of the Peripheral Drift Illusion, with stepwise rather than sawtooth luminance profiles (Fig. 1C) and fragmented or curved edges rather than straight edges (Fig. $1 D, E)$. The resultant Rotating Snakes are characterized by the periodic placement of colored patches of four different luminances along the circumferences of concentric circles. This layout generates the perception of rotational motion in the direction that follows the colored patches from black to blue to white to

Received Nov. 21, 2011; revised Feb. 14, 2012; accepted March 7, 2012.

Author contributions: J.0.-M., S.L.M., and S.M.-C. designed research; J.0.-M. performed research; J.0.-M. analyzed data; J.0.-M., S.L.M., and S.M.-C. wrote the paper.

This work was supported by the Barrow Neurological Foundation (S.L.M., S.M.-C.), the Arizona Biomedical Research Commission (S.M.-C.), and the National Science Foundation (Award 0852636 to S.M.-C.). We thank Andrew Danielson for technical assistance and Hector Rieiro and Michael B. McCamy for their comments.

Correspondence should be addressed to Dr. Susana Martinez-Conde, Laboratory of Visual Neuroscience, Division of Neurobiology, Barrow Neurological Institute, 350 W. Thomas Road, Phoenix AZ 85013. E-mail: smart@neuralcorrelate.com.

DOI:10.1523/JNEUROSCI.5823-11.2012

Copyright (C) 2012 the authors $\quad 0270-6474 / 12 / 326043-09 \$ 15.00 / 0$ yellow to black (Fig. $1 E$ ) (Kitaoka, 2005). A grayscale version of the illusion is effective perceptually as well, and drives the responses of directionally selective visual neurons, so long as the luminance relationship between the patches is preserved (Conway et al., 2005).

The Rotating Snakes is a spatiotemporal illusion, in which both spatial layout and temporal stimulation-usually brought about by eye movements (Backus and Oruç, 2005; Conway et al., 2005; Murakami et al., 2006; Beer et al., 2008; Ashida et al., 2010) — are critical to the perception. The specific eye movements and neural mechanisms involved remain controversial, however.

One theory explaining the phenomenon is that contrastinduced latency differences in neural responses and/or neural adaptation trick the brain's motion detectors into responding to the stationary patches as if to actual motion (Faubert and Herbert, 1999; Backus and Oruç, 2005; Conway et al., 2005; Kitaoka, 2006). For instance, latency differences between higher- and lower-contrast patches may generate equivalent neuronal responses in areas V1 and MT to those induced by actual motion (Conway et al., 2005). If this proposal is correct, any "reset" or "refresh" (Faubert and Herbert, 1999) of the retinal image—caused by microsaccades, saccades, blinks, flash, or displacement of the image - should trigger the motion signal.

A second theory is that the temporal and spatial integration of residual motion vectors-due to incorrectly compensated eye movements - causes the perception of illusory motion (Murakami et al., 2006; Beer et al., 2008; Fermüller et al., 2010). According to this proposal, slow ocular drift triggers the motion signal.

Here we presented subjects with the Rotating Snakes Illusion while we measured their oculomotor output-including microsaccades, blinks, and fixational instability/drift—in correlation to their perception (Fig. 2). Our results show a strong quantitative link between microsaccade and blink production and subsequent illusory rotation. 
A

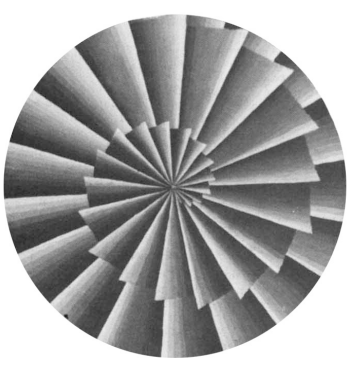

B

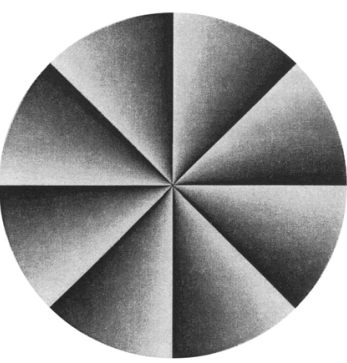

C

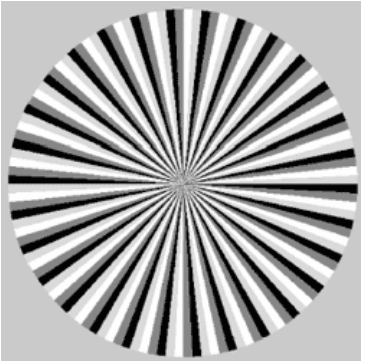

D

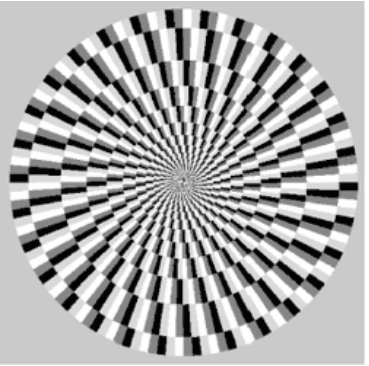

E

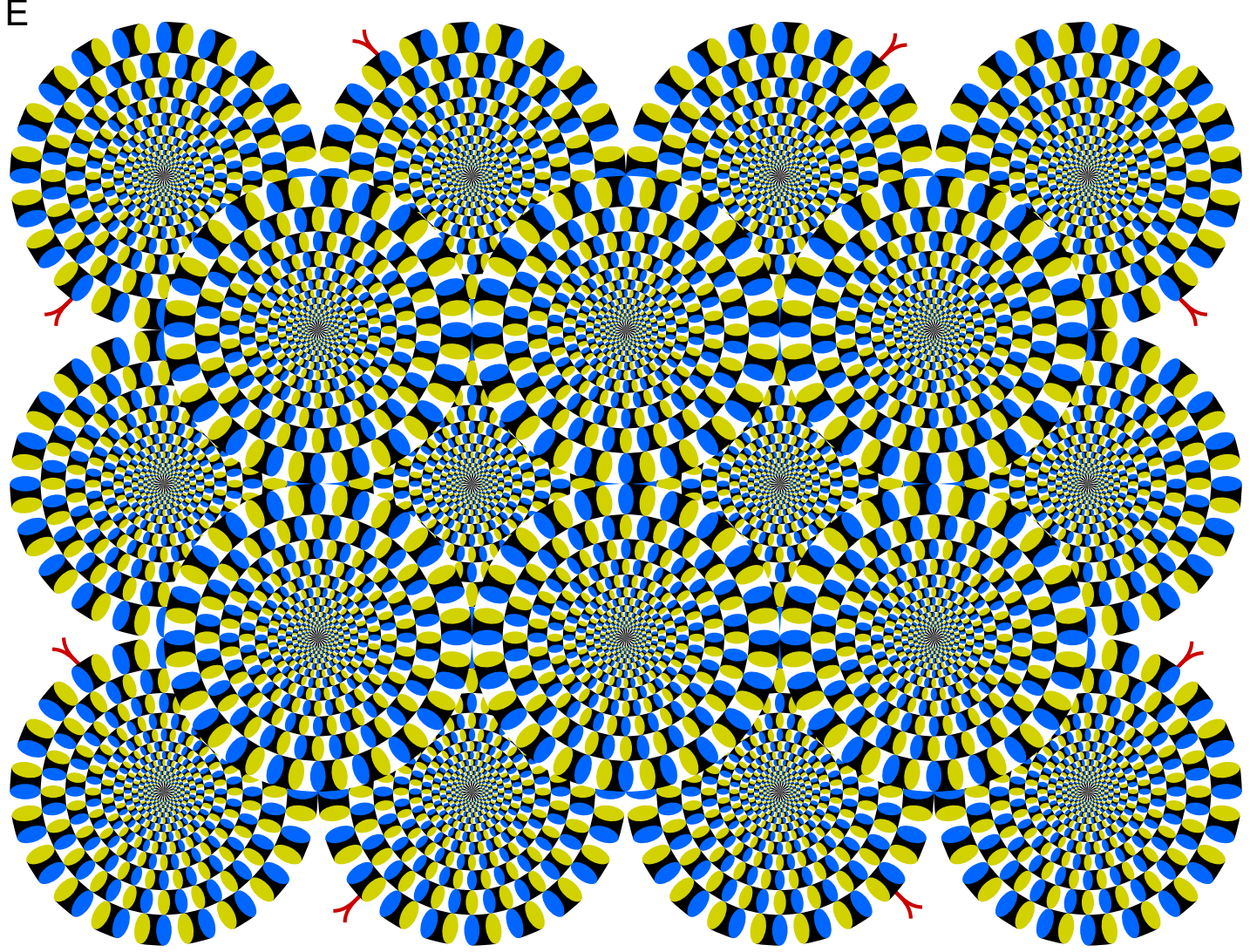

Figure 1. The Rotating Snakes Illusion and its predecessors. A, Fraser-Wilcox Illusion or Escalator Illusion (Fraser and Wilcox, 1979). B, Peripheral Drift Illusion (Faubert and Herbert, 1999). C, The Peripheral Drift Illusion is enhanced by stepwise rather than sawtooth luminance profiles (Kitaoka and Ashida, 2003). D. The Peripheral Drift Illusion is enhanced by fragmented or curved edges (Kitaoka and Ashida, 2003). E, Rotating Snakes Illusion (Kitaoka, 2005).

\section{Materials and Methods}

\section{Subjects}

Eight subjects (6 males, 2 females) with normal or corrected-tonormal vision participated in this study. Each subject participated in three sessions: one training session and two experimental sessions of $\sim 50$ min each. Six of the subjects were naive and were paid $\$ 15$ per session. Experiments were performed under the guidelines of the Barrow Neurological Institute's Institutional Review Board ( protocol 04BN039) and written informed consent was obtained from each participant.

Figures $3 D, 4$, and $5 C, D$ include data from seven subjects only-one subject was excluded due to an insufficient number of transitions toward illusory rotation (i.e., the subject produced only three transitions throughout the whole experiment). Figure 6 includes data from five subjects-three subjects were excluded because of their low blink rates (one or no blinks per trial).
Eye movements recordings and analyses

Eye position was recorded noninvasively in both eyes with a fast videobased eye movement monitor (EyeLink 1000; SR Research) at 500 samples per second (instrument noise, $0.01^{\circ} \mathrm{RMS}$ ).

We identified blink periods as the portions of the EyeLink 1000 recorded data where the pupil information was missing. We added $200 \mathrm{~ms}$ before and after each period to further include the initial and final parts of the blink, where the pupil is partially occluded. We moreover removed those portions of the data corresponding to very fast decreases and increases in pupil area ( $>20$ units per sample) plus the $200 \mathrm{~ms}$ before and after. Such periods are probably due to partial blinks, where the pupil is never fully occluded (thus failing to be identified as a blink by the EyeLink 1000 software) (Troncoso et al., 2008b).

We identified microsaccades automatically with an objective algorithm (for details using $\lambda=6$, see Engbert and Kliegl, 2003). To reduce the amount of potential noise (Engbert, 2006), we analyzed only binocular microsaccades (that is, microsaccades with a minimum overlap of 

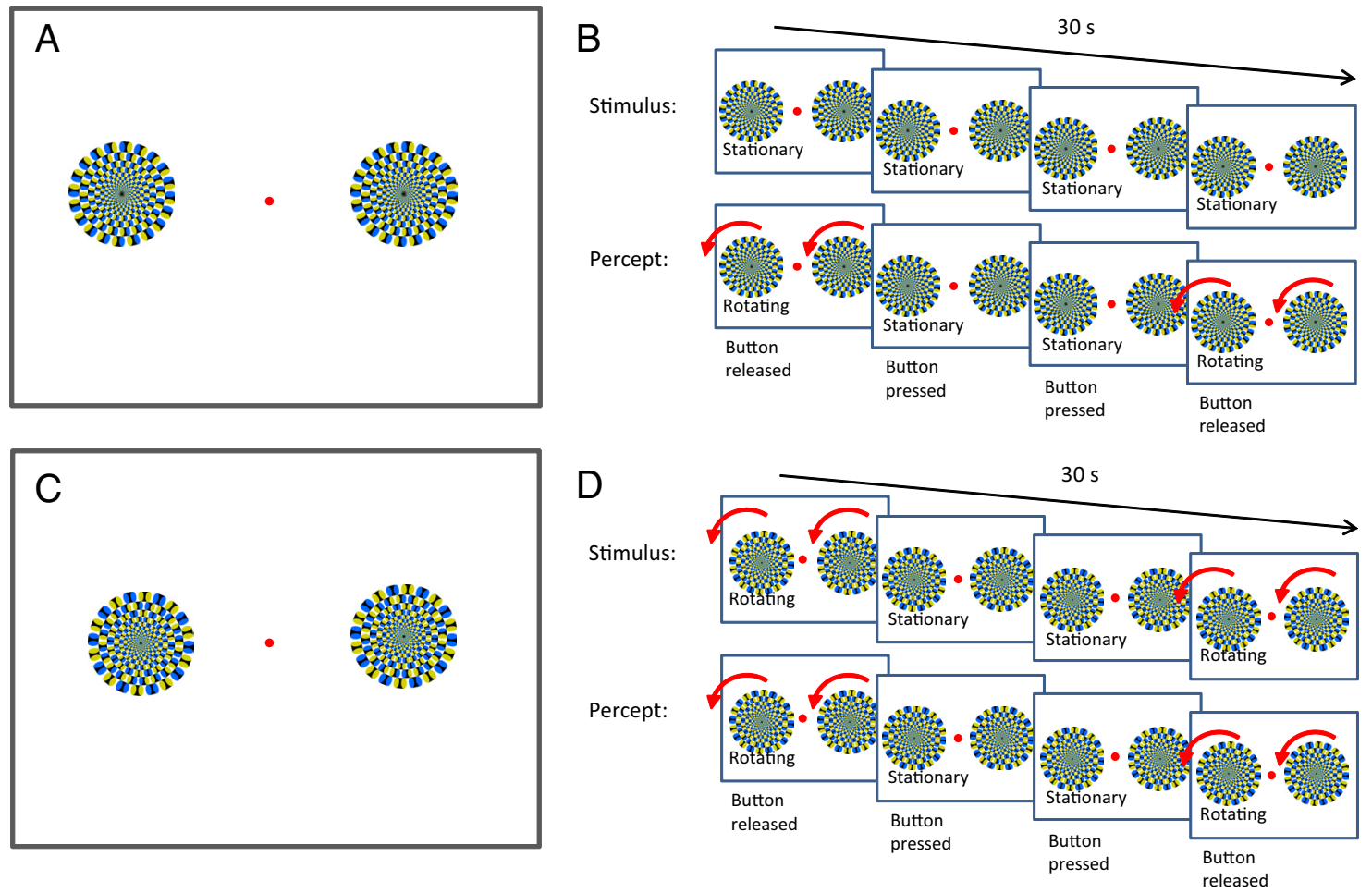

Figure 2. Experimental design. $\boldsymbol{A}$, Set of disks used in the main experimental condition (Rotating Snakes Illusion). The "snakes" appear to rotate. B, An epoch of a trial from the main experimental condition. Top, Schematic representation of the stimulus, which did not change over time. Middle, Perception of the stimulus: the disks intermittently rotated and stopped. Bottom, Subjects' report of their perception. C, Set of disks used in the control condition (physical rotation). The "snakes" appeared stationary. D, An epoch of a trial from the control condition. Top, Schematic representation of the stimulus, which physically rotated and stopped in intermittent fashion. Middle, Perception of the stimulus: subjects identified the stimulus' physical rotation easily. Bottom, Subjects' report of their perception. $A, C$, Fixation dot not to scale. B, D, Fixation dot and "snakes" not to scale.

A

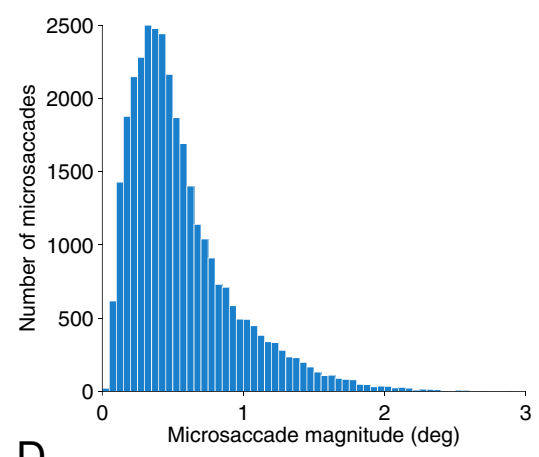

D

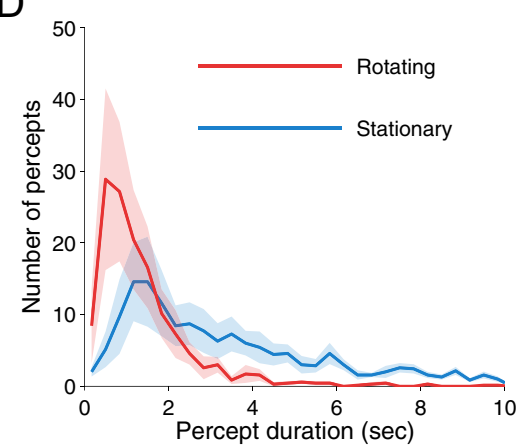

B

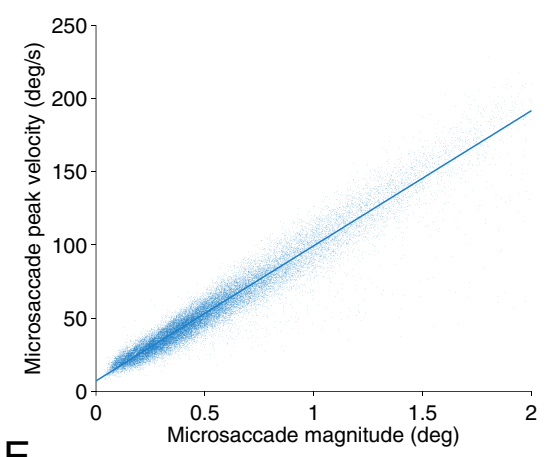

$\mathrm{E}$

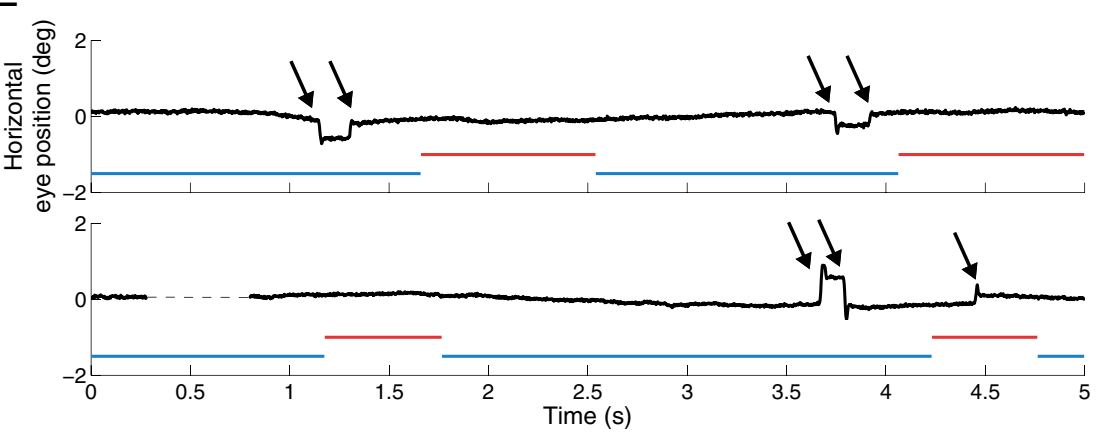

Figure 3. Microsaccade characterization and perceptual reports. $\boldsymbol{A}$, Distribution of microsaccade magnitudes for the subject average ( $n=8$ subjects). $\boldsymbol{B}$, Peak velocity-magnitude relationship for all microsaccades ( $n=23,610$ microsaccades; $n=8$ subjects). C, Distribution of intermicrosaccadic intervals for the subject average ( $n=8$ subjects). $\boldsymbol{D}$, Distribution of durations of rotating (red) and stationary (blue) periods during the main experimental condition, as indicated by the subjects' report ( $n=7$ subjects). Shaded red and blue areas indicate SEM across subjects. $\boldsymbol{E}$, Examples of recorded eye positions in relation to perceptual reports. The gap in the lower eye-position trace, denoted by the dashed line, represents a blink. Arrows identify microsaccades. Drifts are the periods in between microsaccades (or between a blink and a microsaccade). 
one data sample in both eyes) (Laubrock et al., 2005; Engbert, 2006; Rolfs et al., 2006; Troncoso et al., 2008a). We also imposed a minimum intersaccadic interval of $20 \mathrm{~ms}$ so that overshoot corrections were not categorized as microsaccades (Møller et al., 2002; OteroMillan et al., 2008; Troncoso et al., 2008a). One subject produced microsaccades that were often larger than $1^{\circ}$; thus, we chose a maximum microsaccade magnitude threshold of $2^{\circ}$ (Betta and Turatto, 2006; Martinez-Conde et al., 2006; Troncoso et al., 2008a). Across subjects, $>85 \%$ of all microsaccades were smaller than $1^{\circ}$. Figure $3 \mathrm{~A}$ plots the distribution of microsaccade magnitudes for the subject population [up to $3^{\circ}$ (Otero-Millan et al., 2008)]. Of these, we studied further only microsaccades smaller than $2^{\circ}(n=23,610)$ (Troncoso et al., 2008b). Equivalent results were obtained for 1- or 3-degrees-magnitude thresholds (data not shown) (Troncoso et al., 2008b). Figure $3 B$ shows the peak velocity-magnitude relationship for all analyzed microsaccades. Table 1 summarizes various microsaccade and perceptual parameters for individual subjects and for the subject average.

\section{Experimental design}

Subjects rested their head on a chin/foreheadrest $57 \mathrm{~cm}$ from a video monitor (Reference Calibrator V, $60 \mathrm{~Hz}$ refresh rate; Barco). Each experimental session included 48 trials, half of those corresponding to the main experimental condition and the other half corresponding to a control condition, as detailed below. Trials of both types were pseudorandomly interleaved.

Main experimental condition: perception of illusory rotation in the Rotating Snakes Illusion. Subjects fixated on a small red dot $\left(0.25^{\circ}\right.$ in diameter) on the center of the monitor's screen and pressed a key to start each trial. A simplified version of the Rotating Snakes Illusion (including only two $8^{\circ}$-diameter sets of concentric rings, or snake-disks) appeared on the screen upon the key press. The two disks were presented on opposite sides of the screen, with the center of each disk separated from the fixation dot by $9^{\circ}$ (Fig. $2 A$ ). To avoid adaptation across trials, the disk pairs were randomly positioned from one trial to the next, toward one of eight chosen points around the compass $\left(45^{\circ}, 90^{\circ}\right.$, $\left.135^{\circ}, 180^{\circ}, 225^{\circ}, 270^{\circ}, 315^{\circ}\right)$.

Subjects simultaneously and continuously reported whether the disks were stationary (button press) or rotating (button release) (Fig. $2 B$ ). Figure $3 D$ illustrates the distribution of stationary and rotating periods for the subject average, and Table 1 summarizes the corresponding parameters.

After $30 \mathrm{~s}$, all stimuli disappeared and the trial ended. To control for initial stimulusonset transient effects at the start of each trial, we analyzed only those data recorded after the first button press.

Control condition: perception of physical rotation. Here we presented disk pairs that looked very similar to those in the main experimental condition (Rotating Snakes Illusion), but did not generate the perception of illusory motion (Fig. 2C) (Kuriki et al., 2008). These disks rotated physically (0.01 cycles per second), starting and stopping according to
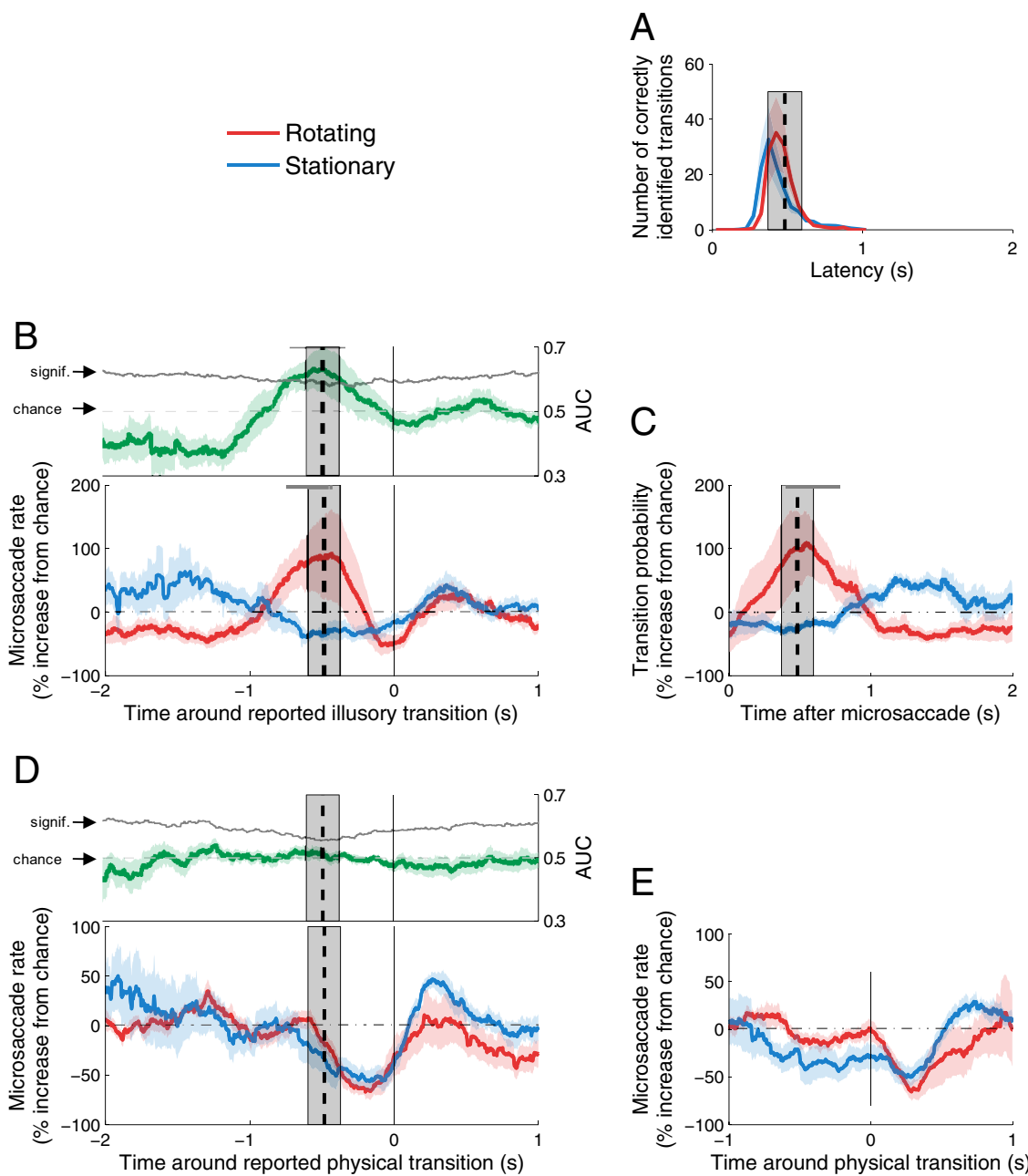

Figure 4. Microsaccades trigger illusory rotation in the Rotating Snakes Illusion. A, Distribution of latencies from correctly identified physical transitions to reported transitions (control condition). Dashed vertical line and surrounding gray bar are the average latency and SD for all subjects $(480 \pm 20 \mathrm{~ms})$. B, C, Perceptual transitions are correlated with previous microsaccades. $\boldsymbol{B}$, Average microsaccade rates around reported transitions toward illusory rotation versus stillness. The vertical gray bar indicates the estimated moment of the perceived (illusory) transitions (mean latency and SD extrapolated from $A$ ). Microsaccade rates depart from baseline $810 \mathrm{~ms}$ before the time of the perceived transitions. The horizontal gray line indicates the bins where microsaccade rates before transitions to rotation were significantly higher than microsaccade rates before transitions to stillness (one-tailed $t$ tests, bin size $=1 \mathrm{~ms}, p \leq 0.01$ ). C, Average probability of transitions after microsaccade onset. The results are expressed as the percentage increase over a correlation where microsaccades happened at random times. $\boldsymbol{D}, \boldsymbol{E}$, Changes in microsaccade rates are not triggered by perceptual transitions. $\boldsymbol{D}$, Average microsaccade rates around reported transitions in physical rotation. Microsaccade rates decreased before reported transitions in physical rotation, regardless of the type of transition (i.e., toward periods of rotation or stillness). The vertical gray bar indicates the moment of the physical transitions with respect to the reported transitions (mean latency and SD from $\boldsymbol{A}$ ). $\boldsymbol{E}$, Average microsaccade rates around physical transitions in rotation. The same data from $\boldsymbol{D}$ are now realigned to the time of the physical transition, rather than to the subjects' report. $\boldsymbol{B}, \boldsymbol{D}$, Insets, Green traces show ROC analysis. The ideal observer can predict the type of illusory transition in the Rotating Snakes Illusion (i.e., toward rotation vs stillness) based on microsaccade rate. $\boldsymbol{B}$, The ideal observer's prediction (green line) becomes significantly better than chance $\sim 660 \mathrm{~ms}$ before the reported transitions (i.e., $\sim 180$ ms before the perceptual transitions; estimated time of perceptual transitions indicated by vertical gray bar; see $\boldsymbol{A}$ ). Horizontal dashed line indicates chance level (probability $\leq 0.5$ ). Thin gray line indicates the significance level at which the ideal observer performs above chance with $p \leq 0.01$ (determined by permutation analysis, see Materials and Methods). Significance is reached whenever the green line is above the gray line. $\boldsymbol{D}$, The ideal observer cannot predict the type of physical transition (i.e., toward rotation vs stillness) for nonillusory rotating objects. Other details are as in $\boldsymbol{B}$, with the horizontal dashed line occluded by the green trace. Each panel shows the results from the subjects' average ( $n=7$ subjects). Shaded red, blue, and green areas indicate the SEM across subjects. AUC, Area under curve.

(i.e., replaying) the timing of the perceptual transitions that each subject had reported in the most recent trial of the main condition. All other details, including the subjects' task (Fig. $2 D$ ), were as in the main condition.

We calculated the average latency between the transitions in physical rotation and the reported transitions (subjects' button presses/releases; 
Table 1. Microsaccade and perceptual parameters

\begin{tabular}{|c|c|c|c|c|c|c|c|c|c|}
\hline & S1 & S2 & S3 & S4 & S5 & S6 & S7 & S8 & Average \pm SEM \\
\hline Number of microsaccades & 1908 & 2690 & 1538 & 2050 & 1143 & 2289 & 2907 & 1619 & $2000 \pm 200$ \\
\hline Microsaccade rate $(N / \mathrm{s})$ & 0.8 & 1.3 & 0.6 & 0.8 & 0.48 & 0.9 & 1.1 & 0.6 & $0.8 \pm 0.1$ \\
\hline Microsaccade magnitude (deg) & 0.39 & 0.44 & 0.5 & 0.23 & 0.6 & 0.47 & 1.0 & 0.6 & $0.5 \pm 0.1$ \\
\hline Microsaccade duration (ms) & 4.9 & 8 & 9 & 4.3 & 12 & 8 & 10 & 6 & $8 \pm 1$ \\
\hline Microsaccade peak velocity (deg/s) & 23 & 26 & 27 & 14 & 27 & 25 & 50 & 30 & $28 \pm 4$ \\
\hline \multicolumn{10}{|l|}{$\begin{array}{l}\text { Main experimental condition: perception of illusory } \\
\text { rotation in the Rotating Snakes Illusion }\end{array}$} \\
\hline Number of transitions to rotation $(N)$ & 72 & 39 & 224 & 3 & 48 & 28 & 257 & 288 & $120 \pm 40$ \\
\hline $\begin{array}{l}\text { Duration of illusory rotation periods (subsequent } \\
\text { to the first illusory period) (s) }\end{array}$ & 1.2 & 2.0 & 1.3 & 0.8 & 3.4 & 0.8 & 0.8 & 1.7 & $1.5 \pm 0.3$ \\
\hline $\begin{array}{l}\text { Duration of first illusory rotation period after } \\
\text { stimulus onset (s) }\end{array}$ & 3.5 & 8.2 & 2.5 & 4.5 & 5.3 & 4.1 & 2.0 & 2.7 & $4.1 \pm 0.7$ \\
\hline Time spent in rotation periods $(\%)$ & 7 & 8 & 22 & 0 & 14 & 2 & 15 & 36 & $13 \pm 4$ \\
\hline Number of transitions to stationary $(N)$ & 69 & 38 & 214 & 3 & 45 & 25 & 253 & 268 & $110 \pm 40$ \\
\hline Duration of stationary periods (s) & 17 & 25 & 4.8 & 410 & 23 & 49 & 4.5 & 3.1 & $70 \pm 50$ \\
\hline Time spent in stationary periods $(\%)$ & 93 & 92 & 78 & 100 & 86 & 98 & 85 & 64 & $87 \pm 4$ \\
\hline \multicolumn{10}{|l|}{ Control condition: perception of physical rotation } \\
\hline Reaction times to transitions to rotating (ms) & 440 & 600 & 390 & 450 & 500 & 500 & 430 & 440 & $470 \pm 20$ \\
\hline Reaction times to transitions to stationary (ms) & 460 & 480 & 440 & 480 & 500 & 600 & 480 & 430 & $490 \pm 20$ \\
\hline Correctly identified transitions to rotating (\%) & 95 & 94 & 98 & 100 & 94 & 78 & 91 & 95 & $94 \pm 2$ \\
\hline Correctly identified transitions to stationary (\%) & 100 & 100 & 97 & 100 & 91 & 90 & 95 & 100 & $97 \pm 1$ \\
\hline
\end{tabular}

S[digit], Subject number.

Fig. 4A). To do this, we discarded latency values $>1500 \mathrm{~ms}[1 \%$ of all reported transitions; such latencies were considered too long to be meaningful (Einhäuser et al., 2008; Troncoso et al., 2008b)]. Table 1 summarizes the corresponding parameters.

\section{Event-triggered averages}

Event-triggered averages (such as those in Fig. 4) were calculated by averaging a multisecond window of data around or after the trigger event [reported transitions (Fig. 4B,D), physical transitions (Fig. 4E), and microsaccade onset (Fig. 4C)]. Thus, the event-triggered average (Fig. $4 B$ ) represents the average percentage increase from chance in microsaccade rate at any given time during the $3 \mathrm{~s}$ window plotted around the reported transitions (all reported transitions are aligned at time $=0 \mathrm{~ms}$ ). If there were $<3 \mathrm{~s}$ of data around a trigger event (due to the edge of the recording time during that specific trial), the $3 \mathrm{~s}$ window was dynamically reduced to fit the available data. For the analyses where the trigger event was a transition, we avoided using the same data point multiple times by considering only the time from the previous transition until the next transition (Einhäuser et al., 2008; Troncoso et al., 2008a).

Individual subjects' event-triggered averages concerning microsaccade rates and transition probabilities were calculated with a $200 \mathrm{~ms}$ sliding window that advanced one data sample $(2 \mathrm{~ms})$ per iteration. For each subject, calculations were made separately for each eye and then averaged between the two eyes. The population average and SEM were then calculated across subjects.

\section{Probability of perceptual transitions triggered by microsaccade onsets}

Only those microsaccades that are produced during a stationary period can trigger the transition to a period of illusory rotation. Conversely, only those microsaccades produced during a period of rotation can trigger a transition to a stationary period. Thus, to calculate the probability of transitions to and from rotating periods as a function of microsaccade onsets, we considered only microsaccades produced in the previous period. To control for chance correlations between microsaccades and transitions, we repeated the above calculations, this time replacing microsaccades with the same number of events, now randomly distributed in time, for each subject. This determined a baseline of chance correlation for each individual subject, from which we calculated the percentage increase in the probability of perceptual transitions after actual microsaccades (Fig. 4C). This analysis is comparable to the cross-correlogram shuffling often used to rule out spurious correlations between potential presynaptic and postsynaptic neurons (Alonso and Martinez, 1998; Martinez and Alonso, 2001).

\section{ROC analysis}

We used a receiver operating characteristic (ROC) analysis (Green and Swets, 1966; Britten et al., 1992; Hernández et al., 2002; Romo et al., 2002, 2004; Feierstein et al., 2006) to quantify how well microsaccade rate predicts the type of perceptual transition (i.e., toward stationary vs rotating "snakes") in the Rotating Snakes Illusion. This analysis makes no assumptions about the underlying probability distributions (Feierstein et al., 2006). The area under the ROC curve provides a measure of the discriminability of two signals and is directly related to the overlap of the two distributions of responses that are compared (Feierstein et al., 2006). In our case, the area under the ROC curve can be interpreted as the probability that an ideal observer, given the microsaccade rate around a particular transition, will correctly determine the type of transition (toward stationary vs toward rotating percepts). An ROC area of 0.5 corresponds to completely overlapping distributions (the ideal observer cannot discriminate between the two types of transition); an area $>0.5$ corresponds to transitions that can be discriminated correctly from the microsaccade rate. At each point in time, we compared the microsaccade rate distributions for transitions toward rotating percepts (true-positive rate) and transitions toward stationary percepts (false-positive rate) in each subject. The ROC curve is the plot of the probability of true positives as a function of the probability of false positives for all possible criterion response levels. We performed a sliding ROC analysis (kernel width 500 $\mathrm{ms}$, slid in $2 \mathrm{~ms}$ increments) to calculate each subject's area under the ROC curve at each time point around the transition (Fig. $4 B, D$ ). To determine the time point at which the ideal observer became better than chance, we calculated significance, using a permutation procedure (Siegel and Castellan, 1988; Hernández et al., 2002; Romo et al., 2002, 2004; Feierstein et al., 2006) with 1000 shuffles for each subject and a criterion level value of $p<0.01$.

\section{Fixation instability analysis}

We measured the variability of ocular drift following Murakami et al. (2006). First, we bandpass (1-31 Hz) filtered the horizontal eye position and calculated the instantaneous eye velocity as the rate of displacement from one data sample to the next. Then, we detected and removed microsaccades (i.e., we used a $10 \%$ s velocity threshold to detect microsaccades and we ignored all data within $\pm 26 \mathrm{~ms}$ around each microsaccade). 

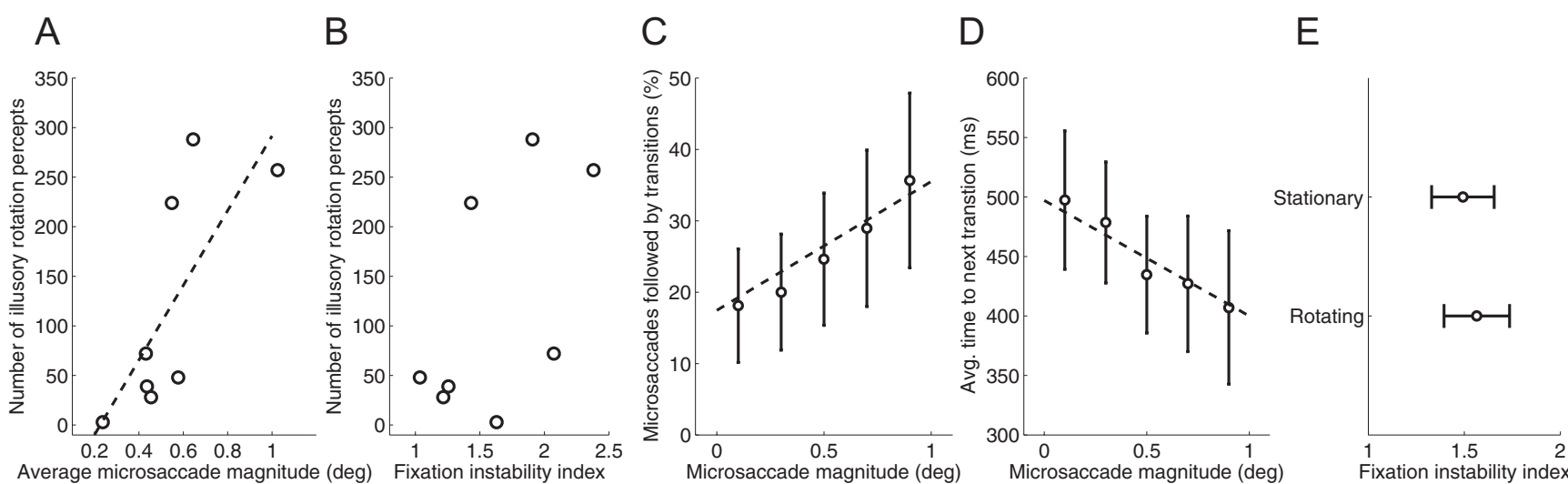

Figure 5. Fixational eye movement parameters and the perception of the Rotating Snakes Illusion. $A$, Correlation between average microsaccade magnitude and number of illusory rotation percepts $(r=0.75, p=0.03)$. B , Correlation between fixation instability and number of illusory rotation percepts $(r=0.58, p=0.14)$, following from Murakami et al. (2006). $\boldsymbol{A}, \boldsymbol{B}$, Each dot represents a different subject $(n=8)$. C, Correlation between microsaccade magnitude and the percentage of times that microsaccades are followed by reported transitions toward illusory rotation (i.e., within the next second). Larger microsaccades resulted in more frequent transitions to illusory rotation than smaller microsaccades $\left(r=-0.54 p=1 \times 10^{-5}\right)$. $\boldsymbol{D}$, Correlation between microsaccade magnitude and latency from microsaccade onset to the next reported illusory rotation. Larger microsaccades resulted in shorter latencies than smaller microsaccades $(r=0.64, p=$ $\left.1 \times 10^{-16}\right)$. E, Fixation instability preceding periods of illusory rotation is equivalent to fixation instability preceding periods of stillness $(p>0.05)$. C, D, Dashed lines show the linear regressions and averages and standard errors are then plotted across subjects $(n=7)$.

Finally, we fit the distribution of the remaining instantaneous eye velocities with a Gaussian and used its standard deviation as the index of fixation instability (Fig. 5B,E). Fixational instability results calculated using the horizontal eye position only (Murakami et al., 2006) were equivalent to those calculated using both horizontal and vertical components (data not shown).

Within-subjects analyses

We performed analysis of covariance, with microsaccade magnitude (bins of $0.2^{\circ}$ ) and subject as independent variables, to calculate the regressions in Figure 5, $C$ and $D$. We calculated the percentage of microsaccades followed by transitions toward rotation within $1 \mathrm{~s}$ of microsaccade onset for each microsaccade magnitude bin and each subject (Fig. $5 C)$. We also calculated the average time from microsaccade onset to the next transition, whenever the next transition occurred within $1 \mathrm{~s}$, for each microsaccade magnitude bin and each subject (Fig. 5D).

We calculated the index of fixation instability during the $1 \mathrm{~s}$ period preceding the transition toward rotation/stillness (for those periods lacking microsaccades and/or blinks) (Fig. 5E).

\section{Results}

Main experimental condition: perception of illusory rotation in the Rotating Snakes Illusion

Subjects fixated a small spot while viewing a simplified version of the Rotating Snakes Illusion (Fig. 2A). They continuously reported (via button press) whether the disks ("snakes") were stationary (button press) or rotating (button release). Their eye movements were recorded simultaneously with high precision (for details, see Materials and Methods, above). Figure $2 \mathrm{~B}$ describes a typical epoch during a trial.

As with other bistable stimuli paradigms (Spillmann and Kurtenbach, 1992; van Dam and van Ee, 2005, 2006a,b; MartinezConde et al., 2006; Troncoso et al., 2008b), subjects reported that their perception oscillated between two alternating states (stillness vs rotation). We found a clear positive relationship between increased microsaccade rates and the perception of snake rotation. Before transitions to a rotation period, microsaccade rates increased (Fig. $4 \mathrm{~B}$, red line). Conversely, before transitions to a period of stillness, microsaccade rates decreased (Fig. $4 B$, blue line). The results were statistically significant (one-tailed $t$ tests; $p \leq 0.01$ ), revealing a direct quantitative link between microsaccade production and the perception of illusory rotation.
To further establish the potential role of microsaccades in driving the Rotating Snakes Illusion, we determined the numbers of transitions to stillness versus rotation periods after microsaccade onsets (Fig. 4C). Microsaccade onsets were followed by increased transitions to rotation and decreased transitions to stillness periods (a 106\% peak increase and a 31\% peak decrease from random, respectively), suggesting a causal relationship between microsaccades and illusory rotation in the Rotating Snakes Illusion. At the very least, we cannot exclude a causal role of microsaccades in driving the Rotating Snakes Illusion, and specifically the perceptual transitions that characterize it. Equivalent results were obtained for naive versus nonnaive subjects, and for maximum microsaccade magnitude thresholds set to $1^{\circ}, 2^{\circ}$, or $3^{\circ}$ (data not shown) (Troncoso et al., 2008b).

\section{Control condition: perception of physical rotation}

We tested an alternative interpretation: that microsaccades do not drive the Rotating Snakes Illusion, but the illusion instead causes both the subjects' reports (button press/release) and the change in microsaccade rate. If this were true, a change in microsaccade rate might precede the subjects' reports (button press/ release), as found in Figure $4 B$, but it would not precede the actual perceptual transitions. To test this possibility, we pseudorandomly interleaved a control condition in which the subjects detected physical rotations for real moving stimuli that simulated the Rotating Snakes Illusion (for details, see Materials and Methods, above; Fig. 2C) (Kuriki et al., 2008). We then used the latency between physical transitions and reported transitions to estimate the timing of perception in the main illusory condition (van Dam and van Ee, 2005, 2006a,b; Troncoso et al., 2008b). Figure $2 D$ describes a typical epoch during a trial. Subjects indicated correctly $95 \pm 2 \%$ of the physical transitions with an average latency of $480 \mathrm{~ms}$ (SD $\pm 100 \mathrm{~ms}$; Fig. $4 \mathrm{~A}$, vertical gray band). The average latency and $\mathrm{SD}$ values were consistent with those reported previously for various bistable stimuli (van Dam and van Ee, 2006a,b; Einhäuser et al., 2008; Troncoso et al., 2008b).

Next, we calculated the rates of microsaccades around both the reported transitions (Fig. 4D) and the physical transitions (Fig. $4 E$; equivalent analyses as in Fig. $4 B$ ). Microsaccade rates decreased before reported transitions to physical stillness as well 
as before reported transitions to physical rotation (a 66\% decrease in microsaccade rate $200 \mathrm{~ms}$ before the reported transition; Fig. $4 D$ ). This is contrary to our findings with illusory rotation: microsaccade rates increased before reported transitions to rotation periods and decreased before reported transitions to stationary periods in the Rotating Snakes Illusion (Fig. 4 B). If perceptual rotation drove microsaccade production, then microsaccade rates should vary comparably for illusory motion (main condition; Fig. $4 B$ ) and physical motion (control condition; Fig. 4D); however, they do not. Thus, perceptual transitions in the Rotating Snakes Illusion do not drive changes in microsaccade rate.

In Figure $4 E$, the data from Figure $4 D$ is realigned around the physical (rather than the reported) transitions. Microsaccade rates decreased after physical transitions, regardless of whether the change was toward rotation or stillness. This result is consistent with previous studies showing that changes in peripheral stimulation cause a transient drop in the rates of saccades and microsaccades, followed by a temporary enhancement (Engbert and Kliegl, 2003; Galfano et al., 2004; van Dam and van Ee, 2006b; Troncoso et al., 2008b). This transient course of microsaccadic inhibition may indicate a fast reflex of the oculomotor system to sudden changes in visual input (Laubrock et al., 2005).

The control condition provided us with the latency between changes in physical rotation and their associated reported transitions (Fig. 4A). This latency, when applied to the Rotating Snakes Illusion results, allowed us to estimate the timing of perceptual transitions due to changes in illusory rotation with respect to reported transitions. To accomplish this, we overlaid the same latency from Figure $4 A$, vertical gray band, on the data from the Rotating Snakes Illusion condition (Fig. $4 B$ ). The results showed that changes in microsaccade rate preceded the perception of the Rotating Snakes Illusion: microsaccade rates began changing (i.e., departed from baseline) $\sim 130 \mathrm{~ms}$ before the perceptual transitions and $810 \mathrm{~ms}$ before the reported transitions in illusory rotation. Thus, microsaccades may trigger the perceptual transitions in illusory rotation, but not the other way around.

Figure $4 C$, moreover, shows that reported transitions to illusory rotation following microsaccades have the same approximate latency $(\sim 500 \mathrm{~ms})$ as reported transitions following actual physical rotation (Fig. $4 A$, gray band). Thus, perceptual transitions following microsaccades are equivalent in their timing to perceptual transitions following physical rotations.

To quantify our conclusions further, we conducted a ROC analysis (for details, see Materials and Methods, above) to calculate the ability of an ideal observer to predict the type of transition (to rotation vs to stillness) based on microsaccade rates (Fig. $4 B, D$, insets, green trace). In the main experimental condition (Rotating Snakes Illusion), the ideal observer becomes significantly better than chance (determined by permutation analysis) $\sim 180$ ms before the estimated perceptual transitions and $\sim 860$ $\mathrm{ms}$ before the reported illusory transitions (Fig. $4 B$, inset, green trace). In the control condition (physical rotation), the ideal observer cannot distinguish between physical transitions to rotation versus to stillness from microsaccade rates alone (Fig. $4 D$, inset, green trace).

We also found microsaccade magnitude to be correlated to the perception of the illusion. Subjects with larger microsaccades perceived illusory rotation more frequently than those with smaller microsaccades (Fig. 5A). Fixation instability did not correlate significantly with illusory perception, suggesting that slow drift does not trigger the perception of the Rotating Snakes Illusion (Fig. 5B).

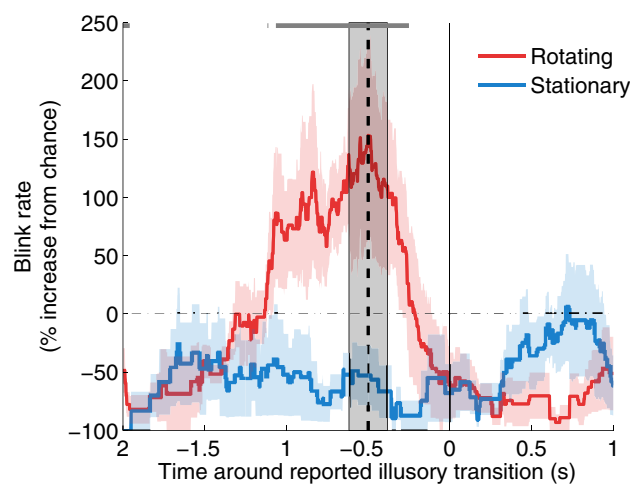

Figure 6. Blinks trigger illusory rotation in the Rotating Snakes Illusion. Average blink rates around reported transitions toward illusory rotation versus stillness. The vertical gray band indicates the estimated moment of the perceived (illusory) transitions (mean latency and SD extrapolated from Fig. $4 A$ ). The horizontal gray line indicates the bins where blink rates before transitions to rotation are significantly higher than blink rates before transitions to stillness (one-tailed $t$ tests, bin size $=1 \mathrm{~ms}, p \leq 0.01$ ).

Within subjects, larger microsaccades led to the perception of illusory rotation more frequently than smaller microsaccades (Fig. 5C) and with shorter latencies (Fig. 5D). In contrast, fixation instability preceding perceived rotation was equivalent to fixation instability preceding stillness (Fig. $5 E$ ) and to fixation instability preceding physical rotation (data not shown).

The perception of the illusion did not vary as a function of the relationship between microsaccade direction and either the angular position of the stimulus or its perceived direction of rotation (i.e., clockwise or counterclockwise). Thus, microsaccades of all directions resulted in the Rotating Snakes Illusion (data not shown).

The results above are consistent with the hypothesis that any significant transient change in the retinal image (i.e., due to microsaccades, saccades, blinks, flashes, or displacement of the image) will trigger a motion signal and thus the perception of illusory motion (Faubert and Herbert, 1999; Backus and Oruç, 2005; Conway et al., 2005; Kitaoka, 2006). If this idea is correct, it follows that not only microsaccades and saccades, but also eyeblinks should generate illusory rotation. To test this possibility, we performed equivalent analyses for blinks as those performed with microsaccades (Fig. 4) and found increased blink rates before transitions to illusory rotation (Fig. 6), thus confirming the predictions from the microsaccade findings and supporting the idea that any transient image change can drive the Rotating Snakes Illusion.

\section{Discussion}

The Rotating Snakes Illusion produces the perception of intermittent illusory rotation. Previous studies investigating the role of eye movements on the Rotating Snakes Illusion (or the related Peripheral Drift Illusion) did not examine its intermittent characteristics, however, focusing instead in the overall strength of the illusion over a given period of time (Murakami et al., 2006; Beer et al., 2008). Here we determined the onset times of intermittent rotation and stillness periods as experienced by observers, then correlated them to microsaccade and blink production.

Microsaccades generate strong neural transients in the early visual system (Martinez-Conde et al., 2000, 2002, 2009) and counteract visual fading during fixation (Martinez-Conde et al., 2006; Troncoso et al., 2008a). They are also correlated to the perception of certain types of illusory motion (Laubrock et al., 2008; Troncoso et al., 2008b). The present results suggest that 
microsaccades (and other transient ocular events such as saccades and blinks) trigger the motion signal underlying the perception of the Rotating Snakes Illusion.

\section{Origin of the motion signal and the role of microsaccades in the perception of the Rotating Snakes Illusion}

The inability of motion sensor units to distinguish between temporal changes in contrast and actual motion can result in the perception of illusory motion (Heeger, 1987). Thus, the Rotating Snakes Illusion and the related Peripheral Drift Illusion may result from abrupt changes in the stimulus (i.e., due to sudden onset or displacement of the image, or to transient ocular events such as microsaccades, saccades, and blinks) leading to contrast-driven latency differences (Faubert and Herbert, 1999). In agreement with this idea, Conway et al. (2005) showed that pairs of stimuli of different contrasts can generate motion signals (i.e., phi movement) in directionally selective neurons of areas V1 and MT.

Our results reveal a tight correlation between microsaccade and blink onsets and the time of the perception of the Rotating Snakes Illusion, supporting the hypothesis that "resetting" or "refreshing" the retinal image triggers the motion signal and the resultant illusory motion (Faubert and Herbert, 1999; Backus and Oruç, 2005; Conway et al., 2005; Kitaoka, 2006). These findings are also consistent with the known physiological and perceptual effects of microsaccades and blinks, such as the generation of neural transients in visual neurons (Gawne and Martin, 2000, 2002; Martinez-Conde et al., 2000, 2002, 2009) and the reversal of visual fading due to adaptation during fixation (Martinez-Conde et al., 2006; Troncoso et al., 2008a; McCamy et al., 2011).

\section{Role of fixation instability in the perception of the Rotating Snakes Illusion}

Previous studies did not find a correlation between microsaccade production and the perception of illusory rotation (Murakami et al., 2006; Beer et al., 2008). The discrepancy between past and present results may be due to differences in experimental design. Murakami et al. (2006) found that subjects with more fixation instability experienced the Rotating Snakes Illusion more strongly than subjects with less fixation instability. They proposed that the illusion results from a failure to compensate the image motion induced by fixational eye movements-particularly ocular drift—with the brain interpreting the residual (uncompensated) motion as actual rotation. Beer et al. (2008) similarly found that fixation instability enhanced the perception of a variant of the Peripheral Drift Illusion. These previous studies presented their stimuli for very short time intervals (i.e., ranging from a few seconds to less than a second), however (Murakami et al., 2006; Beer et al., 2008). It follows that the stimulus onsets themselves may have caused neural transients, resulting in perception of the illusion throughout the entire duration of the trial. Moreover, because microsaccades typically occur once or twice per second, they may have been absent in approximately half of the trials in previous experiments $(\mathrm{Mu}-$ rakami et al., 2006). Therefore, the correlations between fixation instability and illusion strength reported in prior research cannot be disentangled from unmeasured correlations between the stimulus onsets and illusion strength. Accordingly, an alternative interpretation of the previous findings is that fixation instability does not trigger the illusion, but rather modulates its strength once the illusion has been triggered by the stimulus onset itself.

To test the possibility that stimulus onsets could produce illusory percepts lasting for the entire duration of a $1-2 \mathrm{~s}$ trial, we compared the duration of the first illusory period after stimulus onset to the duration of subsequent illusory periods in our data (i.e., throughout our $30 \mathrm{~s}$ trials). The onset-driven illusory period persisted for an average of $4 \mathrm{~s}$, that is, $2.5 \mathrm{~s}$ more than in subsequent illusory periods within the same trial. The difference was significant ( $p=0.005, t$ test) and consistent across individual subjects (Table 1), supporting the idea that stimulus onset is a strong contributor to the illusion and suggesting that previous studies could have conflated the effect of the transient onset with that of fixation instability. The present experiments controlled for this potential confound for the first time by analyzing only the data recorded after the first illusory rotation report (i.e., discarding the only illusory period due to the stimulus onset).

To sum up, the current experiments-by presenting longduration trials and focusing on the illusion's intermittent features (i.e., alternating periods of perceptual rotation vs stillness)—allowed the optimal identification of the oculomotor trigger mechanisms of the Rotating Snakes Illusion. Thus, the lack of correlation between fixation instability and illusory rotation in our data suggest that drift, by itself, cannot trigger the illusion (though it may modulate the strength of the illusion, once triggered). In support of this idea, Backus and Oruç (2005) found that saccade-like jumps in the Rotating Snakes pattern produced illusory rotation, whereas small jitter of the image was ineffective. Similarly, Tomimatsu et al. (2010) found that fast displacements of the image produced a strong illusory percept, whereas smooth motion resulted in a much weaker illusion.

The present findings are in agreement with the growing literature concerning the effects of microsaccades on illusory perception (Martinez-Conde et al., 2006, 2006; Laubrock et al., 2008; Troncoso et al., 2008b; Hsieh and Tse, 2009), particularly those concerning motion illusions (Laubrock et al., 2008; Troncoso et al., 2008b).

\section{Conclusion}

We show a strong quantitative link between microsaccade and blink production and the perception of the Rotating Snakes Illusion during fixation, suggesting that transient ocular events such as microsaccades, saccades, and blinks trigger the perception of illusory rotation. Our results support the theory that visual transients drive the illusory motion signal in the Rotating Snakes Illusion, and suggest that previous reports indicating that either fixation instability or ocular drift trigger the illusion conflate the effects of stimulus onsets with the effect of drift.

\section{References}

Alonso JM, Martinez LM (1998) Functional connectivity between simple cells and complex cells in cat striate cortex. Nat Neurosci 1:395-403.

Ashida H, Kuriki I, Murakami I, Kitaoka A (2010) fMRI adaptation to anomalous motion in the "Rotating Snakes" patterns. J Vis 10:827.

Backus BT, Oruç I (2005) Illusory motion from change over time in the response to contrast and luminance. J Vis 5:1055-1069.

Beer AL, Heckel AH, Greenlee MW (2008) A motion illusion reveals mechanisms of perceptual stabilization. PLoS One 3:e2741.

Betta E, Turatto M (2006) Are you ready? I can tell by looking at your microsaccades. Neuroreport 17:1001-1004.

Britten KH, Shadlen MN, Newsome WT, Movshon JA (1992) The analysis of visual motion: a comparison of neuronal and psychophysical performance. J Neurosci 12:4745-4765.

Conway BR, Kitaoka A, Yazdanbakhsh A, Pack CC, Livingstone MS (2005) Neural basis for a powerful static motion illusion. J Neurosci 25:5651-5656.

Einhäuser W, Stout J, Koch C, Carter O (2008) Pupil dilation reflects perceptual selection and predicts subsequent stability in perceptual rivalry. Proc Natl Acad Sci U S A 105:1704-1709.

Engbert R (2006) Microsaccades: A microcosm for research on oculomotor 
control, attention, and visual perception. Progress in brain research 154:177-192.

Engbert R, Kliegl R (2003) Microsaccades uncover the orientation of covert attention. Vision Res 43:1035-1045.

Faubert J, Herbert AM (1999) The peripheral drift illusion: a motion illusion in the visual periphery. Perception 28:617-621.

Feierstein CE, Quirk MC, Uchida N, Sosulski DL, Mainen ZF (2006) Representation of spatial goals in rat orbitofrontal cortex. Neuron 51:495-507.

Fermüller C, Ji H, Kitaoka A (2010) Illusory motion due to causal time filtering. Vision Res 50:315-329.

Fraser A, Wilcox KJ (1979) Perception of illusory movement. Nature 281:565-566.

Galfano G, Betta E, Turatto M (2004) Inhibition of return in microsaccades. Exp Brain Res 159:400-404.

Gawne TJ, Martin JM (2000) Activity of primate V1 cortical neurons during blinks. J Neurophysiol 84:2691-2694.

Gawne TJ, Martin JM (2002) Responses of primate visual cortical V4 neurons to simultaneously presented stimuli. J Neurophysiol 88:1128-1135.

Green DM, Swets JA (1966) Signal detection theory and psychophysics. Huntington, NY: Wiley.

Heeger DJ (1987) Model for the extraction of image flow. J Opt Soc Am A 4:1455-1471.

Hernández A, Zainos A, Romo R (2002) Temporal evolution of a decisionmaking process in medial premotor cortex. Neuron 33:959-972.

Hsieh PJ, Tse PU (2009) Microsaccade rate varies with subjective visibility during motion-induced blindness. PLoS One 4:e5163.

Kitaoka A (2005) Trick eyes graphics. Tokyo: Kanzen.

Kitaoka A (2006) Anomalous motion illusion and stereopsis. J Three Dimensional Images (Tokyo) 20:9-14.

Kitaoka A, Ashida H (2003) Phenomenal characteristics of the peripheral drift illusion. Vision 15:261-262.

Kuriki I, Ashida H, Murakami I, Kitaoka A (2008) Functional brain imaging of the "Rotating Snakes" illusion. J Vis 8:16.1-16.10.

Laubrock J, Engbert R, Kliegl R (2005) Microsaccade dynamics during covert attention. Vision Res 45:721-730.

Laubrock J, Engbert R, Kliegl R (2008) Fixational eye movements predict the perceived direction of ambiguous apparent motion. J Vis 8:13.1-13.17.

Martinez LM, Alonso JM (2001) Construction of complex receptive fields in cat primary visual cortex. Neuron 32:515-525.

Martinez-Conde S, Macknik SL (2010) The neuroscience of illusion. Scientific Am Special Editions 20:4-7.

Martinez-Conde S, Macknik SL, Hubel DH (2000) Microsaccadic eye movements and firing of single cells in the striate cortex of macaque monkeys. Nat Neurosci 3:251-258.

Martinez-Conde S, Macknik SL, Hubel DH (2002) The function of bursts of spikes during visual fixation in the awake primate lateral geniculate nucleus and primary visual cortex. Proc Natl Acad Sci U S A 99:13920-13925

Martinez-Conde S, Macknik SL, Troncoso XG, Dyar TA (2006) Microsaccades counteract visual fading during fixation. Neuron 49:297-305.

Martinez-Conde S, Macknik SL, Troncoso XG, Hubel DH (2009) Microsaccades: a neurophysiological analysis. Trends Neurosci 32:463-475.

McCamy MB, Otero-Millan J, Macknik SL, Yang Y, Troncoso XG, Baer SM, Crook SM, Martinez-Conde, Susana S (2011) The efficacy and contribution of microsaccades. Society for Neuroscience Meeting, Washington DC, November

Møller F, Laursen ML, Tygesen J, Sjølie AK (2002) Binocular quantification and characterization of microsaccades. Graefes Arch Clin Exp Ophthalmol 240:765-770.

Murakami I, Kitaoka A, Ashida H (2006) A positive correlation between fixation instability and the strength of illusory motion in a static display. Vision Res 46:2421-2431.

Otero-Millan J, Troncoso XG, Macknik SL, Serrano-Pedraza I, MartinezConde S (2008) Saccades and microsaccades during visual fixation, exploration and search: foundations for a common saccadic generator. J Vis 8:21.1-21.18.

Ramachandran VS, Rogers-Ramachandran D (2010) A moving experience: illusions that trick the brain. Scientific Am Mind. January/February:2224.

Rolfs M, Laubrock J, Kliegl R (2006) Shortening and prolongation of saccade latencies following microsaccades. Exp Brain Res 169:369-376.

Romo R, Hernández A, Zainos A, Lemus L, Brody CD (2002) Neuronal correlates of decision-making in secondary somatosensory cortex. Nat Neurosci 5:1217-1225.

Romo R, Hernández A, Zainos A (2004) Neuronal correlates of a perceptual decision in ventral premotor cortex. Neuron 41:165-173.

Siegel S, Castellan NJ (1988) Nonparametric statistics for the social sciences, 2nd ed. New York: McGraw-Hill.

Spillmann L, Kurtenbach A (1992) Dynamic noise backgrounds facilitate target fading. Vision Res 32:1941-1946.

Tomimatsu E, Ito H, Seno T, Sunaga S (2010) The "rotating snakes" in smooth motion do not appear to rotate. Perception 39:721-724.

Troncoso XG, Macknik SL, Martinez-Conde S (2008a) Microsaccades counteract perceptual filling-in. J Vis 8:15.1-15.9.

Troncoso XG, Macknik SL, Otero-Millan J, Martinez-Conde S (2008b) Microsaccades drive illusory motion in the enigma illusion. Proc Natl Acad Sci U S A 105:16033-16038.

van Dam LC, van Ee R (2005) The role of (micro) saccades and blinks in perceptual bi-stability from slant rivalry. Vision research 45:2417-2435.

van Dam LC, van Ee R (2006a) Retinal image shifts, but not eye movements per se, cause alternations in awareness during binocular rivalry. J Vis 6:1172-1179.

van Dam LC, van Ee R (2006b) The role of saccades in exerting voluntary control in perceptual and binocular rivalry. Vision Res 46:787-799. 